\title{
Methodology of technological and operational factors accounting in the process of complex optimal design of micro- and nanodevices manufactured using group technologies
}

\author{
Sergey Meshkov ${ }^{1, *}$ \\ ${ }^{1}$ Bauman Moscow State Technical University, 105005 Moscow, Russia
}

\begin{abstract}
The problem of providing key quality indicators (indicators of purpose, manufacturability, reliability) of micro-and nanodevices at the design stage is considered. The limitations imposed by a group technology production on methods ensuring serial production availability and reliability of micro- and nanodevices are described. The design of microand nanodevices methodology that allows to ensure the optimal balance between serial production availability in the given production conditions and time to failure in the specified operation conditions under given restrictions on the device purpose indicators by taking into account the technological and operational factors is described.
\end{abstract}

\section{Problem statement}

The current practice of designing of micro-and nanodevices includes systems engineering, circuit and engineering design, technological process preparation stages. The device structure is determined at the system engineering stage. The electrical circuit and circuit elements parameters that determine the functional parameters of the device $\boldsymbol{Y}$ (purpose indicators) are the results of circuit design stage. The design implementation of circuit solutions and design parameters $\boldsymbol{X}$, which define the required parameters $\boldsymbol{Y}$, are determined at the design stage. The technological route is developed, the parameters of technological operations are determined and the necessary technological equipment is designed at the technological process preparation (TPP) stage.

This methodology does not take into account the fact that the micro- and nanodevices design imply the use of group technology production methods (often planar), if the device construction elements are located on the same substrate. The high correlation of the devices parameters formed simultaneously within the common substrate during the structural elements production operations is a feature of group technologies. On the other hand, the location of similar structural elements on a common substrate is the reason for the high correlation of aging and degradation processes occurring under the influence of destabilizing factors during device operation. This leads to the fact that the existing linear

\footnotetext{
*Corresponding author: sb67241@mail.ru
} 
design procedure does not allow to achieve devices line optimal technological efficiency and reliability indicators.

\section{Scientific and technical background}

One of the most important characteristics of manufacturability is the serial production availability, which is quantitatively described by the yield probability under given production conditions. The problem of ensuring the micro devices serial production availability was described in [1-3] and developed in [4-5] on the example of SHF hybrid integrated circuits (HIC). In these works it was shown that the known methods of ensuring the devices serial production availability (such as methods of complete, incomplete and group interchangeability that operates with constructive tolerance as a tool of ensuring the serial production availability and accuracy) when using group treatment technologies are not operational due to the high correlation of technological errors of devices parameters within a common substrate.

Application of the adjustment procedure in the transition to microsize is associated with a sharp production cost increasing and also may be impossible due to design and technological limitations. The problem of ensuring device reliability in the specified operating conditions is not considered.

\section{Objective}

The aim of this work is to create a methodology of the micro- and nanoscale devices integrated design, which with the use of the existing group production technology, will achieve the optimal linkage of the main device quality indicators (target values, massavailability) under given production conditions and reliability under given operating conditions.

\section{Main part}

The initial concept is based on the proposition that device specified quality indicator assurance instruments (variable parameters) must, on the one hand, allow their independent variation, and on the other hand, be independent from the group technological production process, which is assumed to be fixed and unchanged. These parameters are the values of the design parameters $X$, and, consequently, the purpose indicators $Y$.

The design methodology has an integrated nature under these conditions, because it aimed on creating a device that, on the one hand, is according to the requirements of the technical specification for the purpose indicators, and on the other hand, it has the optimal technological series production cost within the existing technology and failure time under the specified operating conditions. All three groups of instrument quality indicators are interrelated. Moreover, the device y-percentile time to failure can be considered as the probability of falling devices line random purpose indicators into the given constraints (yield probability), determined after operating time $t$.

The tasks of analyzing and ensuring the stated reliability of radio electronic devices are solved traditionally using the sudden failures model. The device reliability is determined by the reliability indicators of its elements, therefore, device reliability $R$ is equal to the multiplication of the reliability of the device elements $R_{\mathrm{i}}$ :

$$
R=R_{1} * R_{2} * \ldots R_{\mathrm{n}} .
$$


This formula is valid in the case if the reliabilities $R_{\mathrm{i}}$ are independent. The condition of $R_{\mathrm{i}}$ independence is satisfied for a device consisting of discrete electronic components. In this case, $R_{\mathrm{i}}$ is the probability of failure-free operation of one element.

The device elements located within the common substrate are exposed to impact of destabilizing factors (high temperature, ionizing radiation, etc.) during operation. These factors accelerate destructive processes in devices structure. All elements located within the substrate are subjected to destabilizing effects simultaneously. This is the reason for the correlation of the degradation rates of various elements structures and hence elements functional parameters. On the other hand, degradation rates correlation of various elements structures on the substrate is caused by the similarity of the physical processes occurring under the influence of external factors and leading to failure. Therefore, the probabilities $R_{\mathrm{i}}$ are not independent in formula (1). Thus, the probability $R$ should be considered as conditional and formula (1) for its calculation is not applicable.

The gradual failures reliability model is more physically and adequate [6-7]. This model uses the aging processes regularities and takes into account their probabilistic nature, representing a sequence of phenomena and events that lead to failure.

The task of reliability ensuring of the micro- and nanodevices line produced with application of group technology set in the following way. The objective function is the probability of performing the specified functions by devices line $P_{\Phi}^{\Perp}(t, \bar{Y}, \bar{\sigma}, \bar{\Delta})$, where $\bar{Y}$ the device purpose indicators vector $\left({ }^{\bar{Y}}=q(\bar{X})\right), \bar{X}$ - the device design parameters vector; $t$ - operating time, $\bar{\sigma}$ - the device design parameters dispersion vector (technological accuracy); $\bar{\Delta}$ - the vector of tolerance limits of device purpose indicators. $P_{\Phi}^{\Pi}$ is determined as:

$$
P_{\Phi}^{\Pi}=\frac{\sum_{i} \Delta P_{\Gamma}^{i} P_{\Phi}^{i}}{P_{\Gamma}},
$$

where $P_{\Gamma}-$ devices yield probability:

$$
P_{\Gamma}=\int_{Y_{M N}}^{Y_{M A X}} f(Y) d Y,
$$

where $f(Y)$ - the joint probability density function of the device purpose indicators.

The illustrations are shown in Fig. 1 and 2, where $Y_{N O M}$ - the device purpose indicator nominal value; $Y_{M I N}, Y_{M A X}$ - tolerance limits $\bar{\Delta}$ of device purpose indicators; $P_{\Phi}^{i}$ - the probability of performing specified functions with a parameter $Y^{i} ; P_{\Phi}^{M I N}$ - minimum tolerance probability of the device performing the specified functions; $P_{\Gamma}^{i}$ - the probability of the parameter $Y^{i}$ falling into the elementary interval $\Delta Y^{i}$.

A function $P_{\Gamma}(t, \bar{Y}, \bar{\sigma}, \bar{\Delta})$ can be used as the target function if the function $P_{\Phi}(Y)$ is not specified.

Therefore:

- Optimization criterion - $\max P_{\Phi}^{\Pi}(t, \bar{Y}, \bar{\sigma}, \bar{\Delta})$ or $\max P_{\Gamma}(t, \bar{Y}, \bar{\sigma}, \bar{\Delta})$.

- Variable parameters - nominal values $\bar{X}$.

- Limitations - $\bar{\Delta}=$ const, $\bar{\sigma}=$ const, $\bar{X} \in O_{k}, \bar{X} \in O_{T}, P_{\Gamma}(t=0) \geq P_{\Gamma}^{\min }$, where $O_{k}, O_{T}$ - design and technology limits. 
The new nominal values $\bar{X}$ or $Y_{\text {OPT }}$, to which the maximum values $P_{\Phi}^{\mathrm{n}}$ или $P_{\Gamma}$ are correspond, are obtained as a result of solving the optimization problem. The graphical interpretation of the solved problem using the criterion $\max P_{\Gamma}(t, \bar{Y}, \bar{\sigma}, \bar{\Delta})$ is shown in Fig. 3 . The graphical representation of the problem is similar if using $\max P_{\Phi}^{\mathrm{n}}(t, \bar{Y}, \bar{\sigma}, \bar{\Delta})$ criterion.

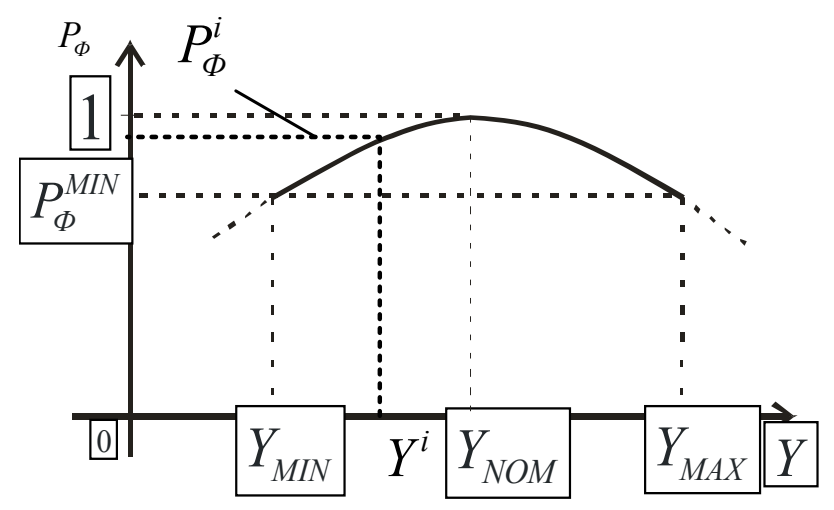

Fig. 1. The dependence of the device performing specified functions probability on the purpose indicator deviation from the nominal value

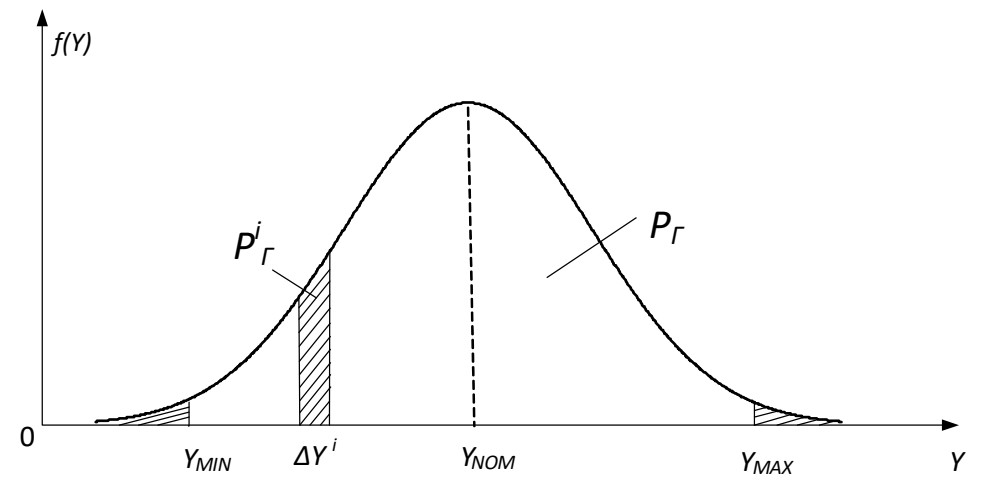

Fig. 2. The devices yield probability determination

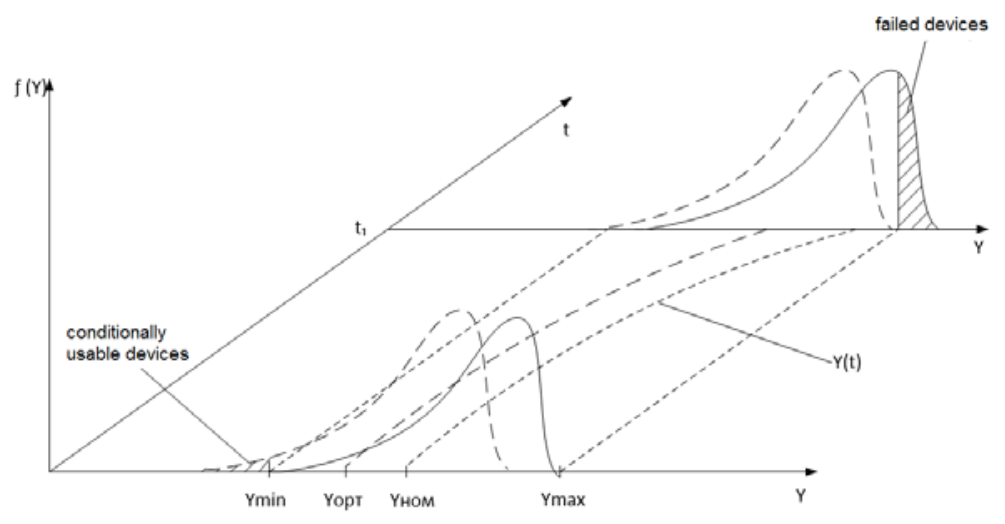

Fig. 3. Graphical representation of the optimization problem using the criterion $\max P_{\Gamma}(t, \bar{Y}, \bar{\sigma}, \bar{\Delta})$ 
This problem is complex, since it allows to maximize the yield probability or the probability of performing specified functions by devices line at $t=0$. Thus, the task of ensuring serial production availability (manufacturability), as well as the task of ensuring reliability (the device's performance indicators must fit the specified requirements) when introducing a time factor and taking into account the device elements degradation process under the influence of external factors are solved.

The scheme of the described methodology is shown in Fig. 4. An additional stage of design and technological optimization is added to the traditional linear design process diagram. At this stage, the parameters $X$ and $Y$ synthesized at the circuit design and engineering design stages are received, as well as technological errors data from the stage of technical preparation of production and data on the design $X(t)$ and functional $Y(t)$ device parameters kinetics under given operating conditions. As a result of design and technological optimization, optimizing corrections to the nominal values $\Delta X_{\text {opt }}$ and $\Delta Y_{\text {opt }}$ (they maximizes the yield) or the probability of performing specified functions by devices line are determined. One of these criteria goes into the category of constraints, when the other is maximized.

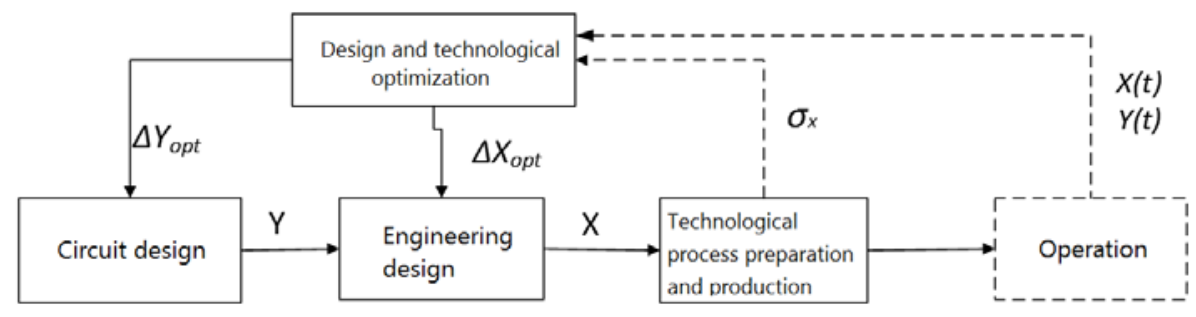

Fig. 4. Complex design methodology scheme.

The problem is solved with the use of simulation methods, including stochastic modeling of technological errors and physical degradation processes of the device elements functional parameters in the specified operating conditions.

The described methodology was developed on the example of micro-and nanoelectronics devices - SHF radio signals mixer and rectifier, in which the resonanttunnelling diode (RTD) was used as a nonlinear element. The methodical basis was the results of the research of the RTD degradation processes under the influence of temperature factor and ionizing radiation, carried out by the authors [8-12], and the RTD diagnostic model based on these results [13-14].

The described methodology approbation shows that, the yield can be increased by 10 $15 \%$, and y-percentile time to failure - by $10-30 \%$ in specified operating conditions by means of optimization of the device design parameters with the unchanged technological process and the purpose indicators conformance with the specifications.

\section{Conclusions}

Group production technology of micro-and nanodevices are characterized by a high correlation of technological errors of the device design parameters. This does not allow, within the conventional design methodology, to ensure high requirements for the serial production availability and reliability indicators without adjusting the existing production technology.

The methodology of complex design is proposed, that allows to provide the optimal balance between serial production availability and reliability in the specified operation 
conditions under given restrictions on the device purpose indicators within the existing technologies.

The research work was supported by Ministry of Education and Science of the Russian Federation under state task № 16.1663.2017/4.6.

\section{References}

1. I.P. Bushminsky, A.G. Gudkov, V.F. Dergachev et al, Design and technological bases of strip microcircuits design (Radio and communication, Moscow, 1987)

2. I.P. Bushminsky, A.G. Gudkov, V.F. Dergachev, SHF circuits design (Bauman University Publishing House, Moscow, 1991)

3. I.P. Bushminsky, G.V. Morozov, SHF circuits technological design (Bauman University Publishing House, Moscow, 2001)

4. A.G. Gudkov, S.A. Meshkov, Machine-builder 12 10-16 (2008)

5. A.G. Gudkov, V.V. Popov, GIC and MIC reliability and quality increasing (Autotest LLC, Moscow, 2012)

6. A.S. Chekanov, Calculations and ensuring the reliability of electronic equipment (KNORUS, Moscow, 2012)

7. A.S. Pronikov, Parametric reliability of machines (Bauman University Publishing House, Moscow, 2002)

8. M.O. Makeev, Y.A. Ivanov, S.A. Meshkov, Semiconductors 50 83-88 (2016)

9. M.O. Makeev, S.A. Meshkov, Y.A. Ivanov, Key Engineering Materials 724 48-52 (2017)

10. M.O. Makeev, S.A. Meshkov, AIP Conference Proceedings 1858020001 (2017)

11. M.O. Makeev, Y.A. Ivanov, S.A. Meshkov et al, 2015 5th International Workshop on Computer Science and Engineering: Information Processing and Control Engineering, WCSE 2015-IPCE. 260-265 (2015)

12. M.O. Makeev, S.A. Meshkov, V.Yu. Sinyakin, J. Phys.: Conf. Ser. 917092004 (2017)

13. M.O. Makeev, S.A. Meshkov, V.Yu. Sinyakin, J. Phys.: Conf. Ser. 741012176 (2016)

14. M.O. Makeev, S.A. Meshkov, V.Yu. Sinyakin, MATEC Web Conf. 12903019 (2017) 